\title{
ANÁlisis EXPERIMENTAl del EFECTO de la tURbULENCIA EN la VElocidad de caída de SEdIMENTOS EN SUSPENSIÓN
}

\section{EXPERIMENTAL ANALYSIS OF TURDULENCE EFFECT IN SETTLING VElOCITY OF SUSPENdEd SEdIMENTS}

\author{
H. Salinas-Tapia, J.A. García-Aragón y C. Díaz-Delgado \\ Centro Interamericano de Recursos del Agua, Facultad de Ingeniería \\ Universidad Autónoma del Estado de México \\ E-mails: hst@uaemex.mx,jgarcia@uaemex.mx,cdiaz@uaemex.mx
}

(Recibido: mayo de 2005; aceptado: junio de 2007)

\section{Resumen}

Por medio de la técnica de Velocimetría por Imágenes de Partículas (PIV), y mediante la aplicación de filtros discriminadores, se calcularon las velocidades de caída de sedimentos para diferentes intervalos de tamaños. Se empleó un canal exper i mental de sección $10 \rtimes 15 \mathrm{~cm}$ y dos valores de caudal líquido con diferentes características de turbulencia para evaluar el efecto de esta última en la velocidad de caída de las partículas de sedimento. La técnica permitió determinar la velocidad de partículas individuales, así como las características del campo de flujo del fluido. La captura y análisis de imágenes se realizó con cámaras digitales tipo CCD por medio del soft ware Sharp-provision PIV, empleando la técnica estadística de correlación cruzada. Los resultados mostraron que la velocidad de caída de las partículas es afectada por la turbulencia, la cual tiende a aumentar el coeficiente de arrastre del fluido. La explicación física del fenómeno se relaciona con la magnitud de la velocidad fluctuante en la vertical del fluido. Sin embargo, se requiere continuar con la inves- tigación para llegar a proponer expresiones de cálculo de la velocidad de caída de sedimentos que tomen en cuenta ese efecto.

Descriptores: Sedimentos, turbulencia, velocidad de caída, PIV, coeficiente de arrastre, tamaño de partícula.

\footnotetext{
Abstract

Set tling ve loc i ties of sed iment par ticles for different size ranges were mea sured in this work us ing PIV with the help of dis crim ina tory fil ters. An experimen tal chan nel 10×15 $\mathrm{cm}$ cross sec tion was used in or der to ob tain two set of tur bu lent char ac ter is tics corre spond ing with two different flow rates. The pur pose was to an a lyze the ef fect of tur bu lence on the sol ids set tling ve loc ity. The tech nique al lowed us to mea sure the in di vid ual set tling ve loc ity of the par ti cles and the flow ve loc ity field of the fluid. Cap ture and im age anal y sis was performed with dig $i$ tal cam eras (CCD) us ing the soft ware Sharp-provision PIV and the sta tis ti cal cross cor re la tion tech nique. Re sults showed that set tling ve loc ity of par ti cles is af fected by tur bu lence which en hances the fluid drag co ef ficient. Phys i cal ex pla na tion of this phe nom e non is re lated with the mag $n+$ tude of the ver tical fluc tu at ing ve loc ity of the fluid. How ever, more re search is needed in or der to de fine set tling ve loc ity for mu las that takes into ac count this ef fect.
} 
Key words: Sediments, turbu lence, settlingvelocity, PIV, dragcoefficient, particlesize.

\section{Introducción}

El análisis del transporte de mezclas de sedimentos en suspensión tiene importantes aplicaciones en el campo de la ingeniería de ríos y embalses. Los resultados pueden igualmente aplicarse en el campo de tratamiento de aguas residuales y en procesos industriales, tanto para el transporte de sólidos finos como para reactores de lecho fluidizado. En general, las mezclas de sedimentos presentan una gran variedad de tamaños. En la práctica ingenieril, para calcular la tasa de transporte de sólidos se ha considerado el diámetro medio de la mezcla y el empleo de fórmulas clásicas (como la de Stokes) y empíricas (como la de Rubey (Yang, 1996)), para calcular la velocidad de caída media de la mezcla. Algunos métodos han considerado intervalos de tamaño de sedimentos para mejorar las predicciones (García-Aragón et al., 2000) pero no consideran el efecto de modificación del arrastre del fluido en movimiento sobre la velocidad de caída de las partículas. La presente investigación pretende determinar este efecto al utilizar dos valores del número de Reynolds del flujo, así como diferentes tamaños de partículas.

La turbulencia afecta el coeficiente de arrastre de las partículas, específicamente con la componente vertical de la velocidad fluctuante. Está comprobado el efecto del número de Reynolds de las partículas sobre el coeficiente de arrastre (Oseen, 1927; Dou, 1963) para el caso de partículas sedimentando en líquidos en reposo. Cuando el líquido está en movimiento, la turbulencia genera velocidades en la dirección vertical que afectan la velocidad de caída de las partículas (Field, 1968; Murray, 1970; Hetsroni, 1981). Sin embargo, un método analítico para cuantificar ese efecto y que sea del conocimiento de los autores del presente trabajo, no se ha presentado en la literatura científica.

En este artículo se pretende explicar el aumento del coeficiente de arrastre para diferentes inter- valos de tamaño de partícula, con la disminución del número de Reynolds de la misma, a causa de la velocidad fluctuante vertical del flujo.

Para tal efecto, se utilizaron mezclas de sedimentos de tamaños cuasi-uniformes vertidas superficialmente en un canal de transporte de sedimentos. Una vez uniformizado el flujo de sedimentos se realiza la captura de imágenes digitales en una sección profusamente iluminada del canal por medio de una hoja de luz láser. Las cámaras sincronizadas con el láser y controladas por medio de una computadora capturan las imágenes digitales en períodos de tiempo muy cortos. Con ayuda del software Sharp-Provision PIV, se analizaron las imágenes, y a partir del desplazamiento de cada partícula y del tiempo entre imágenes, se calcula la velocidad de las partículas. Este procedimiento conocido como Velocimetría por Imágenes de Partículas (PIV) permite calcular la velocidad de caída para cada tamaño de partícula analizado.

\section{Metodología}

Los experimentos se realizaron considerando condiciones de flujo estacionario en un canal para transporte de sedimentos de $2 \mathrm{~m}$ de largo y $10 \mathrm{x}$ $15 \mathrm{~cm}$ de sección. Se utilizaron dos valores de caudal líquido, $0.43 \mathrm{l} / \mathrm{s}$ y $1.48 \mathrm{l} / \mathrm{s}$, así como diferentes diámetros de partículas de sedimentos.

Para medir y calibrar la velocidad del fluido se emplearon como trazadores partículas de poliamida de $25 \mu \mathrm{m}$ de diámetro. Los diámetros de las partículas de sedimentos analizados variaron entre $0.075 \mathrm{~mm}$ y $0.425 \mathrm{~mm}$ (Tabla 1). Para la medición de los campos de velocidades se utilizó un sistema de PIV que consiste en un láser pulsado del tipo Nd-Yag, de doble cavidad, marca NEW WAVE, una cámara digital tipo CCD de marca IDT de alta resolución (1036 x 1360 píxeles) para la captura de las imágenes. La cámara antes mencionada, está provista de un lente de $50 \mathrm{~mm}$ NIKKOR, y se utiliza para capturar un promedio de 
100 imágenes por segundo. En la presente investigación, se capturaron en promedio, 100 pares de imágenes para diferentes intervalos de tamaño de sólidos (Tabla 1) con separación de 2, 5 y 10 ms entre cada imagen.

El sembrado de las partículas, se realizó en forma superficial y cerca de la zona de captura. Para la captura y análisis de las imágenes, se empleó el software de marca Sharp-proVision PIV, que es parte integral del sistema, además ayuda a la sincronía entre la cámara y la fuente láser. La técnica estadística de análisis empleada para el procesamiento de las imágenes, fue la de corre- lación cruzada. La figura 1 presenta el proceso de la técnica PIV.

La captura del primer grupo de imágenes se realizó con frecuencia de pulso de luz láser de 2 ms. El conjunto de imágenes complementarias fue capturado con frecuencia de pulso de 5 y $10 \mathrm{~ms}$ y un promedio de 20 pares de imágenes por corrida con cinco repeticiones por grupo de sedimentos. Una vez capturadas, se realizó la combinación de las imágenes por pares, empleando el software Photo Shop Pro, considerando los canales RGB (Red, Green y Blue), con la finalidad de realizar el análisis por medio de correlación cruzada. En la

Tabla 1. Intervalos de tamaños de partículas de sedimentos

\begin{tabular}{lccc}
\hline & Intervalo 1 & Intervalo 2 & Intervalo 3 \\
\hline Amplitud $(\mathrm{mm})$ & 0.075 a 0.15 & 0.15 a 0.3 & 0.3 a 0.425 \\
Diámetro medio $(\mathrm{mm})$ & 0.1125 & 0.225 & 0.3625 \\
\hline
\end{tabular}

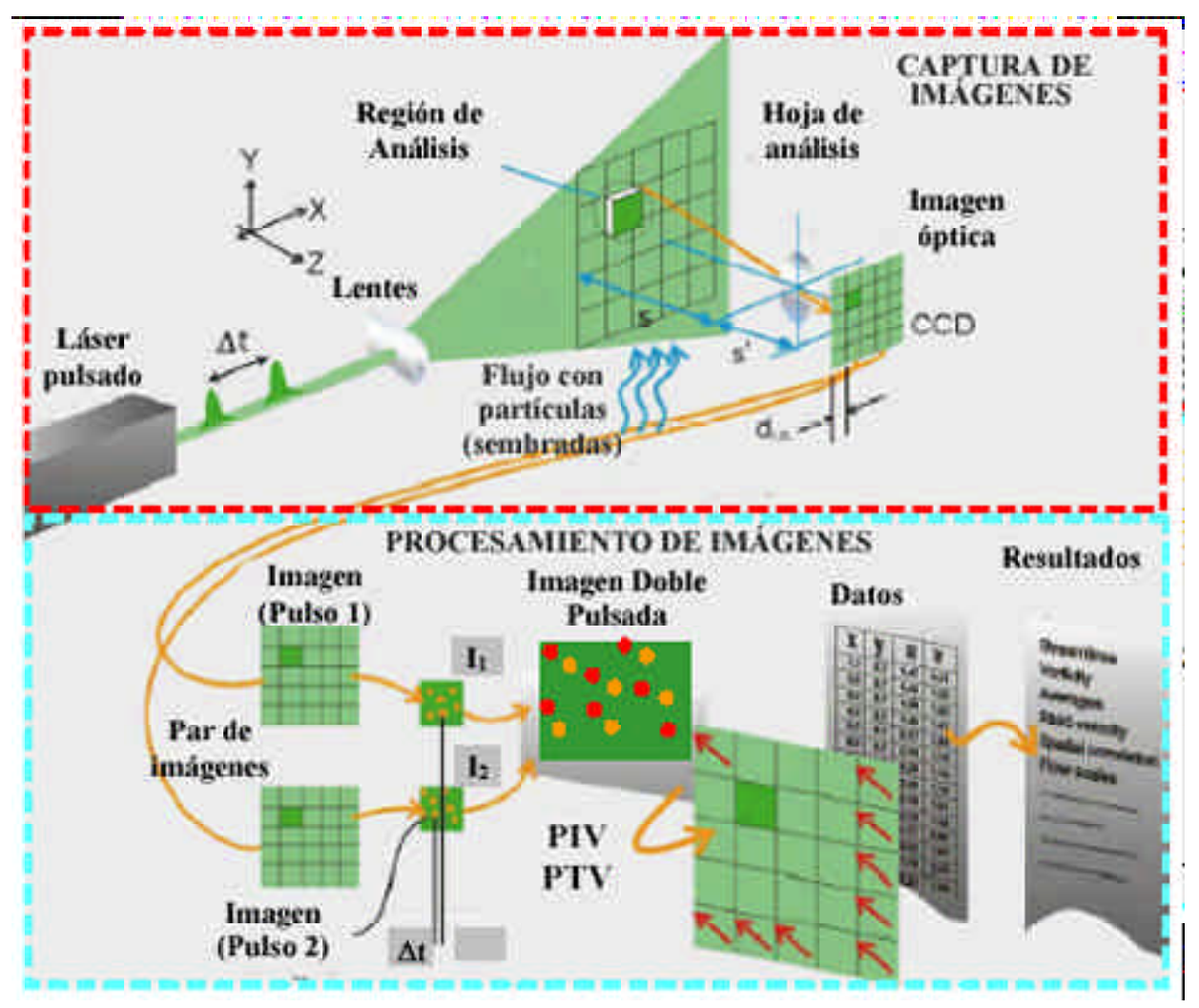

Figura 1. Montaje experimental 
figura 2, se muestra la combinación de dos imágenes capturadas en diferentes pulsos de luz, en esta combinación el canal rojo (R) corresponde a la imagen capturada en el primer pulso, el canal azul (B) corresponde a la imagen capturada en el segundo pulso. En este trabajo se consideró la misma imagen para los canales $\mathrm{G}$ y $\mathrm{B}$, debido a que solamente se tiene la secuencia de dos imágenes. Sin embargo, si se cuenta con imágenes capturadas en pulsos sucesivos, cada canal (RGB) puede corresponder a una imagen distinta con periodos de pulso iguales o diferentes. En el análisis de las imágenes se aprecian claramente los pares de partículas; no obstante, por tratarse de un intervalo de tamaño de partículas, éstas presentan formas y tamaños diferentes.

\section{Resultados}

Se realizaron diferentes corridas para determinar el área de interés (análisis) óptima, para la determinación de los vectores de velocidad. Se analizaron áreas de $32 \times 32$ píxeles, con sobreposición (overlap) del 50 y $75 \%$, de 64 x 64 píxeles y de
128 ×128 píxeles, con el mismo overlap. El área de análisis que presentó mejores resultados, es decir, la menor cantidad de vectores erróneos fue la de $64 \times 64$ píxeles con overlap del 50\%. Por lo que, para esta combinación, y para cada par de imágenes se determinaron un promedio de 1300 vectores, de los cuales, en promedio, 100 vectores resultaron erróneos.

En las figuras 3 y 4, se presenta el valor de la esperanza matemática de los campos de velocidad para el grupo de partículas correspondiente a la muestra con intervalos de diámetros entre 0.15 a $0.30 \mathrm{~mm}$. Estos campos de velocidad se presentan para los valores de 4250 y 14800 del Reynolds del flujo $\left(\operatorname{Re}_{\mathrm{f}}=u h / \mathrm{v}\right)$, correspondientes a las dos condiciones de flujo analizadas. Estas condiciones son; velocidades promedio $(u)$ de $8.5 \mathrm{~cm} / \mathrm{s}$ y de $17.4 \mathrm{~cm} / \mathrm{s}$ y tirantes (h) de $5 \mathrm{~cm}$ y $8.5 \mathrm{~cm}$, respectivamente. Como se puede apreciar, la velocidad de caída de las partículas varía de acuerdo con la profundidad y horizontalmente en vista de que existe variedad de tamaños de partículas.

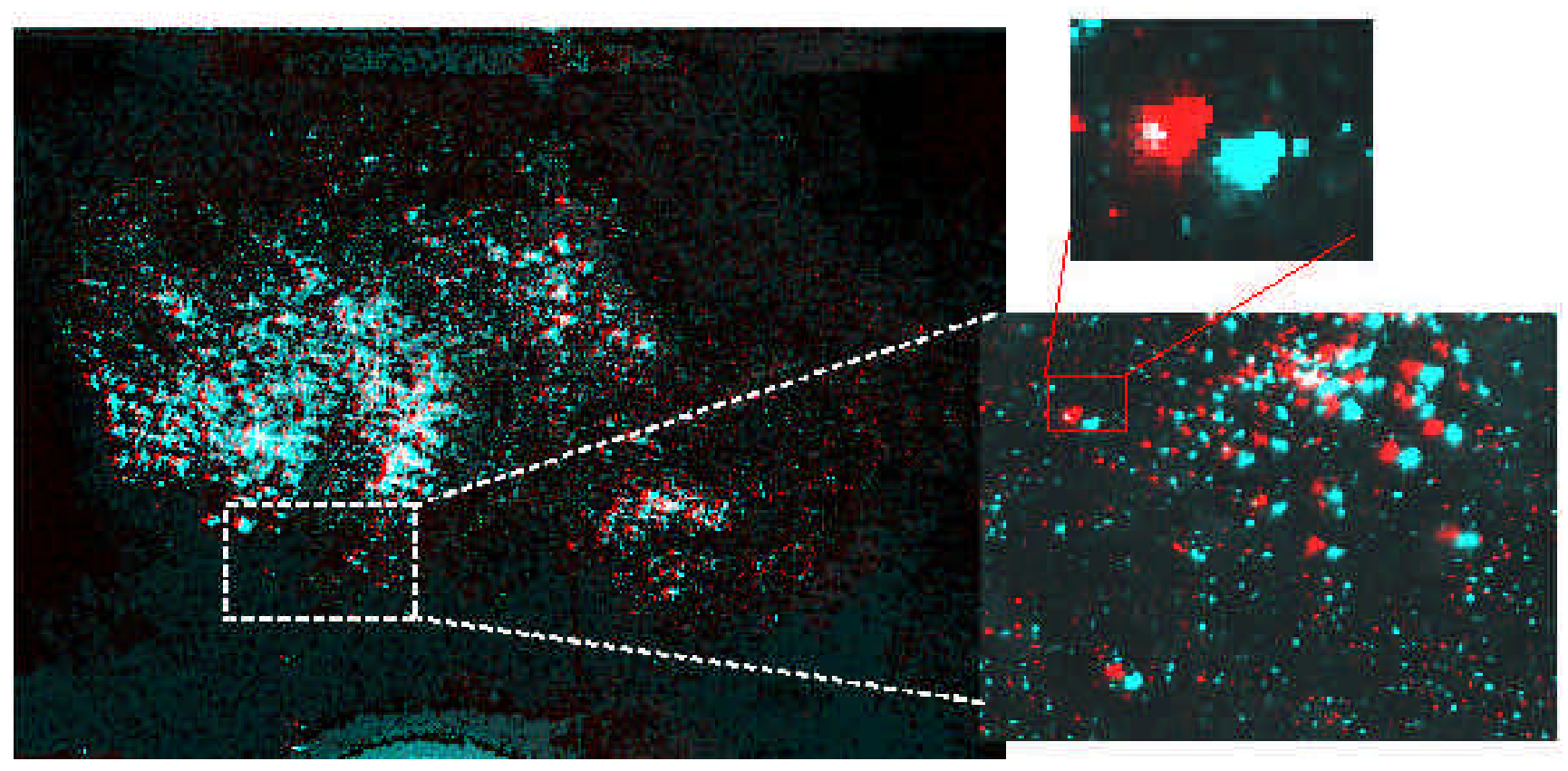

Figura 2. Imagen capturada para sedimentos del intervalo de 0.075 a $1.150 \mathrm{~mm}$ de diámetro 


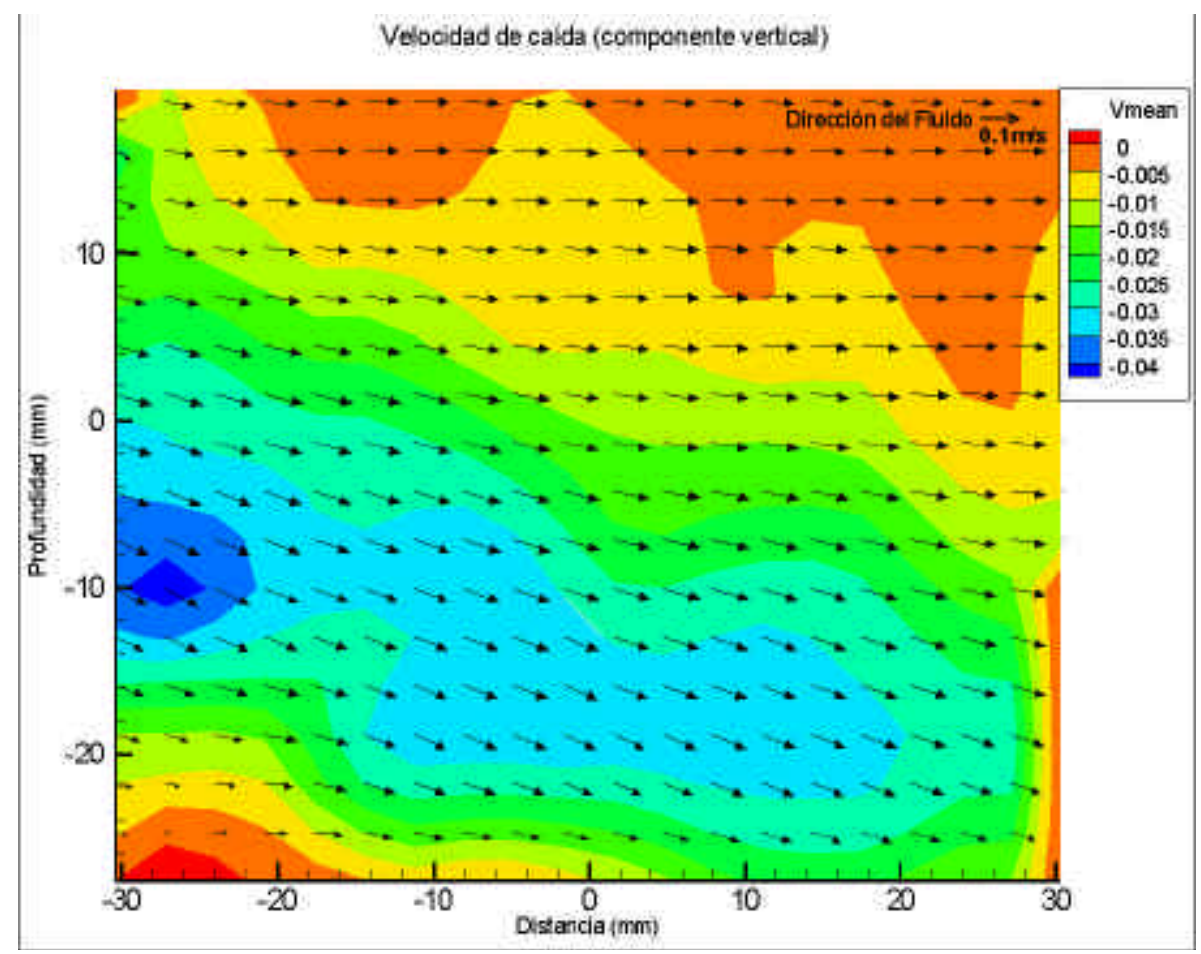

Figura 3. Campos de velocidad promedio para sedimentos del intervalo $0.15-0.30 \mathrm{~mm}$, $R e_{f}=4250, u=8.5 \mathrm{~cm} / \mathrm{s}, ?{ }_{s}=3.25 \mathrm{~cm} / \mathrm{s}$

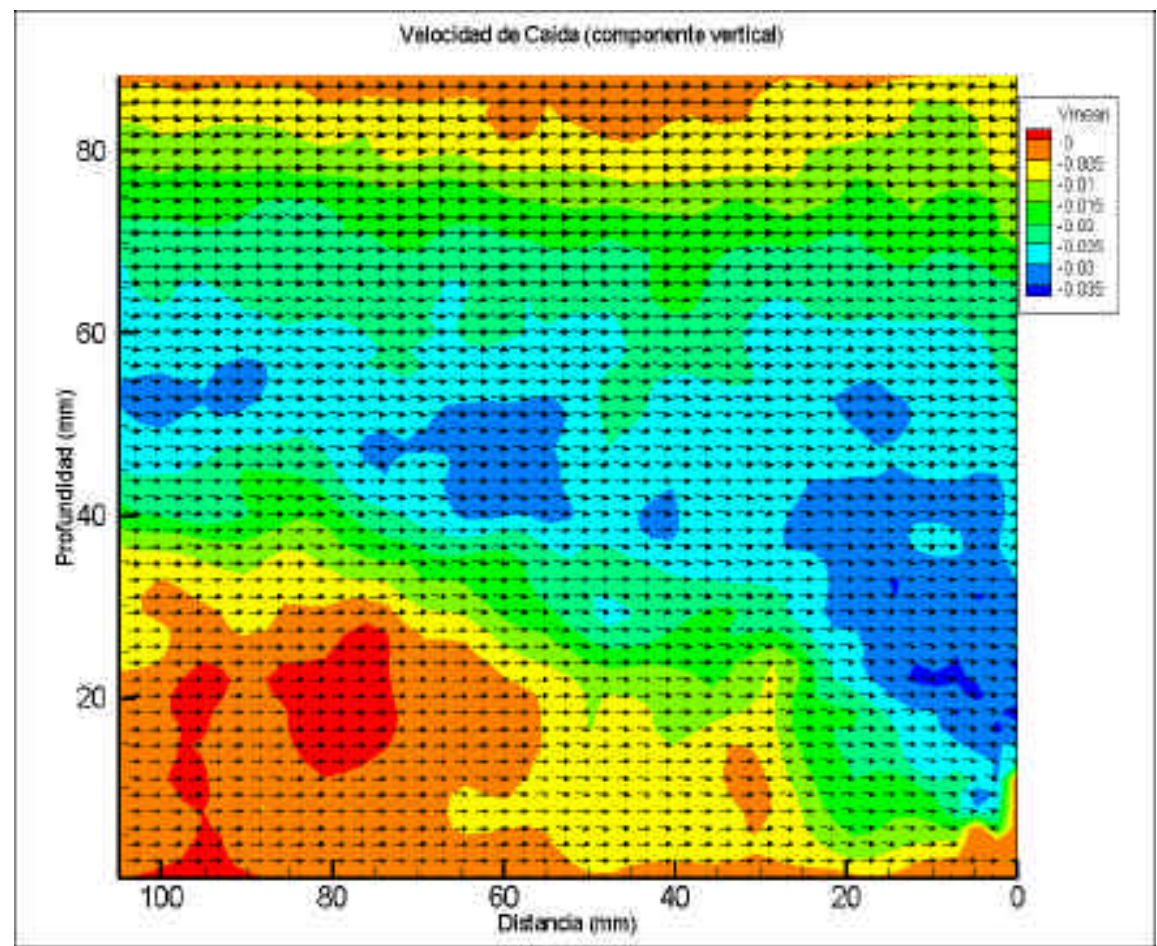

Figura 4. Campos de velocidad promedio para sedimentos del intervalo $0.15-0.30 \mathrm{~mm}$, $R e_{f}=14800, u=17.4 \mathrm{~cm} / \mathrm{s}, ?_{s}=3.19 \mathrm{~cm} / \mathrm{s}$ 
Los valores medios obtenidos para la velocidad de caída ? s de cada uno de los tres intervalos de tamaño de partícula analizados se presentan en la tabla 2.

Con el objetivo de determinar la influencia de la turbulencia en la velocidad de caída de las partí- culas, se calculó la componente en la vertical de la velocidad fluctuante del fluido $\left(V^{\prime}\right)$. Las figuras 5 y 6 muestran los resultados para los dos números de Reynolds del flujo analizados y en la tabla 3 se encuentran los valores promedio calculados para cada uno de los mismos.

Tabla 2. Valores experimentales de $\omega_{\mathrm{s}}(\mathrm{cm} / \mathrm{s})$, para diámetro medio de los intervalos

\begin{tabular}{ccc}
\hline$d_{\text {promedio }}$ & $\operatorname{Re}_{f}=4250$ & $\operatorname{Re}_{f}=14800$ \\
\hline$(\mathrm{mm})$ & $\omega_{s}(\mathrm{~cm} / \mathrm{s})$ & $\omega_{s}(\mathrm{~cm} / \mathrm{s})$ \\
0.1125 & 0.62 & 0.48 \\
0.225 & 1.75 & 1.62 \\
0.3625 & 3.25 & 3.19 \\
\hline
\end{tabular}

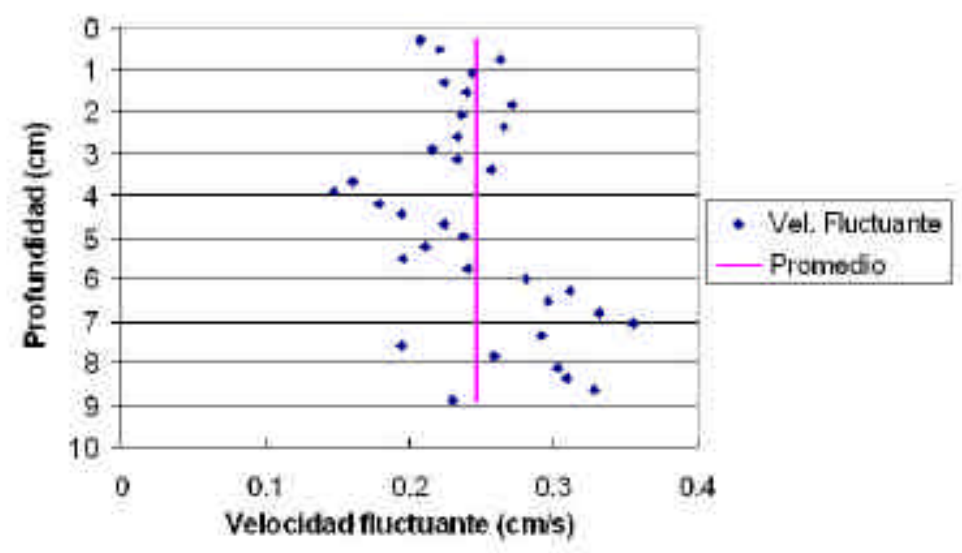

Figura 5. Velocidad fluctuante en la vertical ( $v^{\prime}$ ) para $R e_{f}=14800$

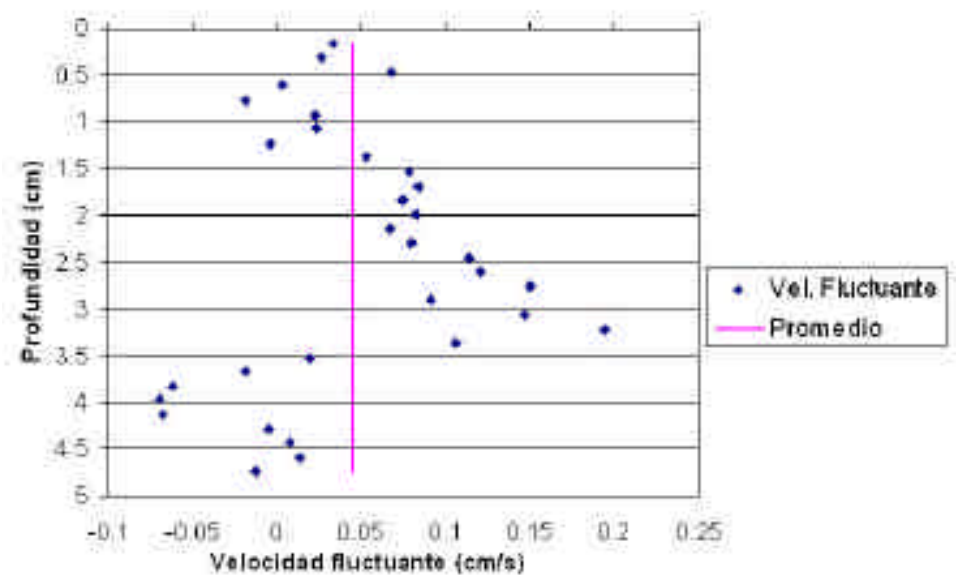

Figura 6. Velocidad fluctuante en la vertical (v') para $\operatorname{Re}_{f}=4250$ 
Tabla 3. Velocidades fluctuantes medias en la vertical

\begin{tabular}{ccl}
\hline & $\mathrm{U}(\mathrm{cm} / \mathrm{s})$ & $\overline{v^{\top}}(\mathrm{cm} / \mathrm{s})$ \\
\hline $\mathrm{Re}_{f}=4250$ & 8.5 & 0.045 \\
$\mathrm{Re}_{f}=14800$ & 17.4 & 0.25 \\
\hline
\end{tabular}

\section{Discusión}

El parámetro de velocidad de caída $\rho_{s}$ ) produce incertidumbre en los cálculos, puesto que la fórmula de Stokes sólo es válida para la velocidad de caída de una partícula esférica aislada, sedimentando con números de Reynolds de partícula menores a 1. Para el caso general de partículas no-esféricas sedimentando con altos números de Reynolds es necesario usar ecuaciones empíricas. Esas ecuaciones empíricas utilizan diámetros equivalentes promedio de un grupo de partículas. Con base en el análisis de diferentes estudios experimentales para partículas de sedimentos naturales, tales como arenas, Hallermeier (1981) establece tres ecuaciones que describen la relación entre la velocidad de caída y el índice de flotación de Arquímedes, cada ecuación es válida para un cierto número de Reynolds de la partícula $\left(\mathrm{Re}_{\mathrm{p}}\right)$, el cual se define en la ecuación 1.
De forma similar, She et al . (2005) desarrollaron expresiones empíricas para sedimentos naturales. Estas relaciones empíricas se presentan en la tabla 4. Se puede notar, que las ecuaciones originales se encuentran reformuladas conforme al mismo formato del número de Reynolds $\mathrm{Re}_{\mathrm{p}}$, expresado como una función del tamaño de la partícula y a un parámetro adimensional $\left(D_{g r}\right)$, definidos por:

$$
\begin{aligned}
& \operatorname{Re}_{\rho}=\frac{\omega_{s} d}{v} \\
& D_{g r}=d \sqrt[3]{\frac{g(s-1)}{v^{2}}}
\end{aligned}
$$

donde ? es la velocidad de caída de la partícula $(\mathrm{m} / \mathrm{s}) ; d$ es el diámetro de la partícula $(\mathrm{m}) ; v$ es la viscosidad cinemática del fluido $\left(\mathrm{m}^{2} / \mathrm{s}\right) ; \mathrm{s}$ es la densidad relativa $\left(\rho_{s} / \rho\right)$ y g la aceleración debida a la gravedad $\left(\mathrm{m} / \mathrm{s}^{2}\right)$.

\begin{tabular}{|c|c|c|c|c|}
\hline Autor & & uación & Notas & No. \\
\hline Ley de Stokes & \multicolumn{2}{|l|}{$\operatorname{Re}_{p}=\frac{D_{g r}^{3}}{18}$} & $\begin{array}{l}\text { 1. Para partículas esféricas } \\
\text { 2. Valida para } D_{g r}<3.42\end{array}$ & 3 \\
\hline \multirow{3}{*}{ Hallermeier (1981) } & $\operatorname{Re}_{p}=\frac{D_{g r}^{3}}{18}$ & $\left(D_{g r} \leq 3.42\right)$ & $\begin{array}{l}\text { 1. La primera de las tres ecuaciones es la } \\
\text { misma de Stokes }\end{array}$ & 4 \\
\hline & $\operatorname{Re}_{p}=\frac{D_{g r}^{2.1}}{6}$ & \multicolumn{2}{|l|}{$\left(D_{g r}=3.42-21.54\right)$} & 5 \\
\hline & $\operatorname{Re}_{p}=1.05 D_{g r}^{1.5}$ & $\left(D_{g r} \geq 21.54\right)$ & 2. Para partículas de arena & 6 \\
\hline \multirow[t]{2}{*}{ She et al. (2005) } & \multicolumn{2}{|c|}{$\operatorname{Re}_{p}=1.05 D_{g r}^{1.5}\left[1-e^{-0.08 D_{g r}^{1.2}}\right]$} & 1. Para arenas con $D_{g r}>2$ & 7 \\
\hline & \multicolumn{2}{|c|}{$\operatorname{Re}_{p}=1.05 D_{g r}^{1.5}\left[1-e^{-0.315 D_{g r}^{0.7552}}\right]^{2.2}$} & 2. Para arenas con $D_{g r}<2$ & 8 \\
\hline
\end{tabular}

Tabla 4. Ecuaciones para detrminar la velocidad de caída a partir del número de Reynolds de la partícula 
Para la aplicación de estas fórmulas, es necesario considerar las limitaciones, particularmente al aplicarse a sedimentos naturales, ya que han sido propuestas considerando partículas de diámetro uniforme, en flujos en reposo y sólo un grupo con aplicación para arenas.

Es indispensable caracterizar adecuadamente las partículas en tamaño y forma, de ahí la necesidad de que estos parámetros no sean supuestos, sino medidos de forma real aplicando técnicas ópticas. En la tabla 5, se presentan los valores de la velocidad de caída, empleando las fórmulas empíricas de Hallermeier (1981) y de She et al. (2005), para los tamaños medios analizados y las obtenidas experimentalmente con la técnica PIV.

De lo anterior, se puede deducir que las fórmulas empíricas tienen una diferencia promedio con el valor obtenido para $\operatorname{Re}_{\mathrm{f}}=4250$ de 16\% (la mayor diferencia es para el primer intervalo con $21 \%$ ) y con el valor obtenido para $\mathrm{Re}_{\mathrm{f}}=14800$ de $7.8 \%$ (la mayor diferencia es para el tercer intervalo con 13.7\%). Se observa claramente que para flujos turbulentos, los valores de la velocidad de caída dependen de la intensidad de la turbulencia, ya que existe una tendencia a que esta velocidad disminuya con el aumento del número de Reynolds del flujo, como se observa en la tabla 5 para cada tamaño de partícula.

La explicación física de esta tendencia a disminuir $\omega_{s}$ con el aumento de $\operatorname{Re}_{f}$ responde al aumento de la componente vertical velocidad fluctuante. Para cuantificar este efecto, se utilizó la expresión propuesta por Dou (1963), la cual considera el aumento de la zona de separación de flujo en la estela de la partícula a medida que aumenta $\mathrm{Re}_{p}$. El referido autor define el ángulo de separación $\theta$ en la estela de una partícula esférica con la ecuación

$$
\theta=1.78 \log \left(4 \operatorname{Re}_{p}\right)
$$

El coeficiente de arrastre según Dou, está dado por

$C_{D}=0.45 \sin ^{2} \frac{\theta}{2}+\frac{24}{\operatorname{Re}_{p}}\left[1+\frac{3}{16} \operatorname{Re}_{p}\right] \frac{\left(1+\cos \frac{\theta}{2}\right)}{2}$

considerando los valores de velocidad fluctuante de la tabla 3 y restando esos valores a la velocidad media calculada con las fórmulas de She et al. (2005), se obtuvieron los valores del coeficiente de arrastre según la expresión (10). La tabla 6 muestra los resultados anteriores, donde el parámetro $\mathrm{Re}_{\mathrm{p}}$ ' representa al número de Reynolds de la partícula afectado por la media de velocidad fluctuante vertical y el correspondiente coeficiente de arrastre es $C_{D}$ '.

$$
\operatorname{Re}_{p}=\frac{\left(\omega_{s}-\overline{v^{\prime}}\right) d}{v}
$$

En la tabla 6 se puede observar que el coeficiente de arrastre aumenta de manera pronunciada con el aumento de $R e_{f}$. Por ejemplo, para partículas del primer intervalo (con valor

Tabla 5. Velocidad de caída $\left(\omega_{\mathrm{s}}\right)$ para diámetro medio de los diferentes intervalos

\begin{tabular}{ccccc}
\hline \multirow{2}{*}{$d_{\text {promedio }}(\mathrm{mm})$} & Hallermeir & She et al. & $\mathrm{PIV}\left(\mathrm{Re}_{f}=4250\right)$ & $\mathrm{PIV}\left(\mathrm{Re}_{f}=14800\right)$ \\
\hline 0.1125 & 0.49 & 0.53 & 0.62 & 0.48 \\
0.225 & 1.58 & 1.53 & 1.75 & 1.62 \\
0.3625 & 2.67 & 2.94 & 3.25 & 3.19 \\
\hline
\end{tabular}


medio $d=0.1125 \mathrm{~mm}$ ) hay un $70 \%$ de aumento de $C_{D}{ }^{\prime}$ al aumentar $\operatorname{Re}_{f}$ de 4250 a 14800 . Para partículas mayores el incremento del valor de $C_{D}$ ' es menor, para el segundo intervalo es $10 \%$ y para el tercer intervalo es 3\%.

\section{Conclusiones}

El análisis del transporte de una mezcla de sedimentos cuasi-uniforme en condiciones de variación de flujo líquido y sólido, ha permitido dilucidar efectos de interacción entre el fluido y los sólidos. Estos efectos repercuten en las propiedades del flujo, especialmente en las características de turbulencia y en la velocidad de caída de las partículas. La técnica de Velocimetría por Imágenes de Partículas (PIV) ha permitido determinar las velocidades de caída para diferentes intervalos de tamaño de partículas en suspensión, lo cual es difícil de lograr con otros métodos convencionales como ADV (Velocimetría Acústica Doppler) o LDV (Velocimetría Láser Doppler).

El incremento en las características de turbulencia del flujo, disminuye el número de Reynolds de las partículas. Como consecuencia, el coeficiente de arrastre se ve claramenente aumentado en las partículas menores del caso experimental analizado (intervalo 1-0.075 a 0.15 $\mathrm{mm}$ ), lo cual implica una disminución sensible de la velocidad de caída en la medida en que aumente el $\mathrm{Re}_{\mathrm{f}}$. En el caso del primer intervalo se midió una disminución de la velocidad de caída de $30 \%$ con un aumento de $\operatorname{Re}_{f}$ de 4250 a 14800 .

Finalmente, a pesar de los avances logrados en este trabajo se concluye que es requerida investigación adicional para determinar una relación entre el $\mathrm{Re}_{f}$ y el coeficiente de arrastre de las partículas, con el fin de obtener una forma de calcular la velocidad de caída de las partículas en condiciones de turbulencia del fluido incremetadas.

\section{Referencias}

Dou-Gouren. Theory of sediment transport. Nanjing research Institute of water con servancy. China. 1963. Pp.5.1-5-38.

Field W.G. Effects of density ratio on sedimentary similitude. Journal of Hydraulics di- vision, ASCE, 94 (HY3): 705-719. 1968.

García-Aragón J.A., Salgado-Salazar S., Morales-Reyes P. y Diaz-Delgado C. Determinación de perfiles de concentración de sedimentos en suspensión para granulometría extendida en situaciones de no-equilibrio. Revista Ingeniería Hidráulica en México, (2 II época). Mayo-Agosto 2000.

Hetsroni G. Parti cles-turbulence inter ac tion. Int. J. Multiphase Flows,. 15(5):735-746.1989.

Hallermeier J. Terminal settling velocity of commonly occurring sand grains. Sedimentology, (28):859-865. 1981

Tabla 6. Coeficientes de arrastre afectados por la velocidad fluctuante vertical del fluido

\begin{tabular}{|c|c|c|c|c|c|c|c|c|}
\hline $\mathrm{Re}_{f}$ & $\begin{array}{c}U \\
(\mathrm{~cm} / \mathrm{s})\end{array}$ & $\begin{array}{c}\overline{\bar{v}^{\prime}} \\
(\mathrm{cm} / \mathrm{s})\end{array}$ & $\begin{array}{c}\mathrm{d} \\
(\mathrm{mm})\end{array}$ & $\begin{array}{c}\omega_{\mathrm{s}} \\
(\mathrm{cm} / \mathrm{s}) \\
\end{array}$ & $\mathrm{Re}_{p}$ & $\operatorname{Re}_{p}$ & $C_{D}$ & $c_{D}{ }^{\prime}$ \\
\hline \multirow{4}{*}{4250} & & & 0.1125 & 0.58 & 0.59 & 0.54 & 44.71 & 48.45 \\
\hline & 8.5 & 0.045 & 0.225 & 1.60 & 3.44 & 3.34 & 11.39 & 11.61 \\
\hline & & & 0.3625 & 3.4 & 10.65 & 10.49 & 6.67 & 6.71 \\
\hline & & & 0.1125 & 0.58 & 0.59 & 0.31 & 44.71 & 80.68 \\
\hline \multirow[t]{2}{*}{14800} & 17.4 & 0.25 & 0.225 & 1.60 & 3.44 & 2.88 & 11.39 & 12.76 \\
\hline & & & 0.3625 & 3.4 & 10.65 & 9.75 & 6.67 & 6.88 \\
\hline
\end{tabular}


Murray S.P. Settling velocities and vertical diffusion of particles in turbulent water. Journal of geophysical research, 75(9).1970.

Oseen C.W. (1929). Neueure Methodenand Ergebnisse in der hydrodynamic. Akademische Verlagsgesellschaft, Leipzig, cited by (Yang 1996).
She K., Trim L., and Pope D. Fall Velocity of natural sediment particles: a simple mathematical presentation of the fall velocity law. J. Hydraul. Res,43(2): 189-195. 2005.

Yang C.T. Sediment transport theory and practice. New York, USA. McGraw Hill. 1996.

\section{Semblanza de los autores}

Humberto Salinas-Tapia. Es ingeniero civil por la Facultad de Ingeniería de la Universidad Autónoma del Estado de México desde el año 2000. Durante el mismo año, obtuvo la maestría en ciencias del agua por el Centro Interamericano de Recursos del Agua (CIRA)- Facultad de Ingeniería-UAEM. Actualmente, estudia el doctorado en ingeniería en la Universidad Autónoma del Estado de México (UAEM) y es profesor de asignatura del CIRA-UAEM.

Juan Antonio García-Aragón. Es ingeniero civil por la Universidad de Los Andes, Bogotá, Colombia desde 1980. Obtuvo la maestría en ciencias por la Universidad Laval, Québec, Canada en 1989 y el doctorado en ingeniería civil y mecánica aplicada por la Universidad McGill, Montreal, Canada en 1993. Actualmente es profesor-investigador del Centro Interamericano de Recursos del Agua de la Universidad Autónoma del Estado de México desde 1994 y miembro de la Asociación Internacional de Ingeniería e Investigación en Hidráulica-IAHR desde 1998. Es miembro nivel I del Sistema Nacional de Investigadores y miembro de la Orden de Ingenieros de Québec (OIQ) desde 1996.

Carlos Díaz-Delgado. Es ingeniero civil por la Universidad Autónoma de Querétaro, Querétaro, México (1986). Logró la maestría en ciencias el año de 1988 por la Universidad Laval, Québec, Canada. Asimismo, el doctorado en ingeniería civil por la misma institución, pero en 1992. A la fecha, es profesor-investigador del Centro Interamericano de Recursos del Agua- Universidad Autónoma del Estado de México, desde 1994, así como miembro nivel I del Sistema Nacional de Investigadores y miembro de la Orden de Ingenieros de Québec (OIQ), desde 1994. 